Supplement of The Cryosphere, 13, 1635-1659, 2019

https://doi.org/10.5194/tc-13-1635-2019-supplement

(c) Author(s) 2019. This work is distributed under

the Creative Commons Attribution 4.0 License.

(c) (1)

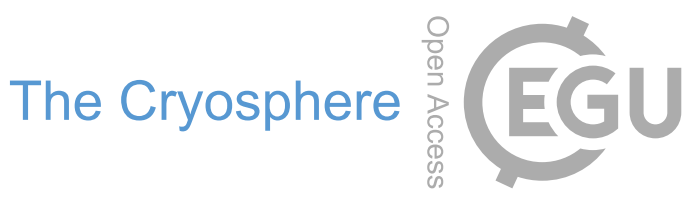

Supplement of

\title{
Warming temperatures are impacting the hydrometeorological regime of Russian rivers in the zone of continuous permafrost
}

Olga Makarieva et al.

Correspondence to: Olga Makarieva (o.makareva@spbu.ru,omakarieva@gmail.com)

The copyright of individual parts of the supplement might differ from the CC BY 4.0 License. 


\section{Tables $\mathbf{S 1}$ - S4 designations}

The cells filled with grey color correspond to statistically significant trends with $\mathrm{p}<0.10$. If any value is bold, it has significance $\mathrm{p}<0.05$; if a value is in italics, it has significance $0.05<\mathrm{p}<0.10$. In Tables 4 (precipitation) and 7-9 (streamflow) each cell with significant trend contains three numbers: 1) the value of total change for the whole period of observations in the characteristic unit (for example, mm) 2) percentage of total change $(\%) ; 3$ ) where available - the year of change point or letter " $\mathrm{m}$ " for monotonical trend. If there is neither year, nor " $\mathrm{m}$ ", the

15 Pettitt's test was not carried out due to too many gaps in the data. Statistically significant trend values are divided into 4 groups and marked with different colors accordingly: change points around 1966 - red, 1970-1985 - green, 1986-1995 - violet, 1996 and later - yellow. Monotonous trends and where change points were not available due to too many gaps in the data are in black. For streamflow, the year of change point marked with * indicates that the gauge has a long-term series of more than 70 years with change point in about 1966 and no significant trend after that period (last 50 years). In some cases, a second year of change point is given in brackets, as estimated with Buishand range test. 
Table S1: Detected changes of monthly and annual air temperature $\left({ }^{\circ} \mathrm{C}\right)$ and change points (year).

\begin{tabular}{|c|c|c|c|c|c|c|c|c|c|c|c|c|c|c|c|}
\hline Index & Period & 1 & 2 & 3 & 4 & 5 & 6 & 7 & 8 & 9 & 10 & 11 & 12 & Avg & ${ }^{*} \mathrm{CPY}$ \\
\hline \multicolumn{16}{|c|}{ Yana River basin } \\
\hline 21931* & $1961-2015$ & 2.8 & 0.7 & 2.9 & 4.1 & 3.1 & 2.0 & 1.4 & 3.0 & 1.7 & 1.8 & 3.0 & 2.1 & 2.2 & 0.40 \\
\hline 24261 & $1966-2012$ & $\begin{array}{l}3.1 \\
\mathrm{~m}\end{array}$ & -3.3 & -0.1 & 1.7 & $\begin{array}{l}3.1 \\
\text { m }\end{array}$ & 2.3 & 2.2 & 2.4 & -0.2 & 0.5 & & -0.1 & $\begin{array}{l}1.4 \\
\mathrm{~m}\end{array}$ & 0.30 \\
\hline $24266^{*}$ & $1969-2015$ & 5.6 & 2.1 & 0.0 & 2.3 & 3.8 & 1.7 & 3.2 & 2.1 & 0.5 & 0.6 & 1.9 & 4.5 & 2.1 & 0.45 \\
\hline 24371 & $1942-2015$ & $\begin{array}{l}3.5 \\
1990\end{array}$ & 0.7 & 1.0 & $\begin{array}{l}3.4 \\
1967\end{array}$ & $\begin{array}{l}3.3 \\
1970\end{array}$ & 0.8 & $\begin{array}{l}1.5 \\
m\end{array}$ & 0.3 & -0.4 & 0.7 & $\begin{array}{l}4.1 \\
1982\end{array}$ & 1.9 & $\begin{array}{l}1.8 \\
1982\end{array}$ & 0.24 \\
\hline \multicolumn{16}{|c|}{ Indigirka River basin } \\
\hline 21946 & $1939-2015$ & 1.4 & 0.4 & 1.4 & 2.8 & 1.1 & 0.4 & $\begin{array}{l}1.5 \\
1986\end{array}$ & & $\begin{array}{l}.9 \\
1979\end{array}$ & $\begin{array}{l}4.4 \\
1993\end{array}$ & $\begin{array}{l}4.7 \\
1993 \\
\end{array}$ & 2.5 & $\begin{array}{l}.3 \\
1987\end{array}$ & 0.30 \\
\hline 24076 & $1960-2015$ & $\begin{array}{l}\mathbf{4 . 6} \\
1992 \\
\end{array}$ & 1.8 & 2.5 & & & 0.9 & 1.8 & $\begin{array}{l}2.0 \\
1994 \\
\end{array}$ & $\begin{array}{l}1.6 \\
\mathrm{~m}\end{array}$ & 1.7 & 3.2 & 3.1 & $\begin{array}{l}2.5 \\
1987\end{array}$ & 0.45 \\
\hline 24382 & $1938-2015$ & $\begin{array}{l}5.1 \\
1975\end{array}$ & $\begin{array}{l}3.0 \\
1978\end{array}$ & $\begin{array}{l}4.1 \\
1983\end{array}$ & $\begin{array}{l}4.5 \\
1980\end{array}$ & $\begin{array}{l}3.5 \\
1987\end{array}$ & 1.1 & $\begin{array}{l}2.2 \\
1986\end{array}$ & 1.4 & 1.3 & $\begin{array}{l}.5 \\
1987\end{array}$ & $\begin{array}{l}6.3 \\
1983\end{array}$ & $\begin{array}{l}5.2 \\
1978\end{array}$ & $\begin{array}{l}3.6 \\
1987\end{array}$ & 0.46 \\
\hline $24585^{*}$ & 1966-2012 & -1.6 & 0.1 & 4.0 & 1.4 & 1.8 & 2.2 & 1.7 & 0.7 & 0.3 & 1.5 & 4.7 & 2.9 & 1.7 & 0.36 \\
\hline 24588 & $1957-2015$ & 2.4 & 0.1 & & 1.0 & 1.2 & 1.4 & $\begin{array}{l}1.6 \\
\mathrm{~m}\end{array}$ & 1.0 & 0 & 2.1 & $\begin{array}{l}3.8 \\
\text { m }\end{array}$ & 2.4 & $\begin{array}{l}1.8 \\
1979\end{array}$ & 0.31 \\
\hline $24679^{*}$ & $1942-2015$ & 1.7 & -0.9 & 2.5 & (1.8 & 2.8 & 1. & 1.0 & -0.6 & -0.7 & 0.5 & 2.3 & 1.2 & 1.2 & 0.16 \\
\hline 24684 & 2015 & 1.3 & -0.3 & & 1.1 & 1.9 & $\begin{array}{l}2.0 \\
1988\end{array}$ & $\begin{array}{l}2.1 \\
1990\end{array}$ & 1.2 & 0.6 & 1.1 & $\begin{array}{l}5.7 \\
1983\end{array}$ & 3.2 & $\begin{array}{l}2.1 \\
1979\end{array}$ & 0.36 \\
\hline 24688 & $1935-2015$ & $\begin{array}{l}3.7 \\
1973\end{array}$ & 0.8 & $\begin{array}{l}4.1 \\
1988\end{array}$ & $\begin{array}{l}2.8 \\
1969\end{array}$ & $\begin{array}{l}.7 \\
1970\end{array}$ & $\begin{array}{l}2.1 \\
1985\end{array}$ & $\begin{array}{l}1.6 \\
1993\end{array}$ & 0.7 & 0.5 & 1.4 & 1.4 & 1.8 & $\begin{array}{l}2.0 \\
1979\end{array}$ & 0.25 \\
\hline 24691 & $1966-2015$ & 1.6 & -0.5 & & 1.3 & 1.9 & 1.4 & $\begin{array}{l}2.2 \\
1987\end{array}$ & 1.0 & 0.3 & $\begin{array}{l}3.1 \\
1993\end{array}$ & $\begin{array}{l}6.2 \\
1983 \\
\end{array}$ & $\begin{array}{l}5.0 \\
1994\end{array}$ & $\begin{array}{l}2.3 \\
1993\end{array}$ & 0.46 \\
\hline
\end{tabular}


Table S2: Detected changes of monthly and seasonal precipitation (mm) and change points (year), 1966-2015.

\begin{tabular}{|c|c|c|c|c|c|c|c|c|c|c|c|c|c|c|c|c|}
\hline Index & Period & 1 & 2 & 3 & 4 & 5 & 6 & 7 & 8 & 9 & 10 & 11 & 12 & Year & Cold (10-4) & Warm \\
\hline \multicolumn{17}{|c|}{ Yana River basin } \\
\hline 21931 & $1966-2015$ & 2.5 & -1.4 & 3.0 & 0.0 & -0.7 & -10.0 & -12.9 & $\begin{array}{l}-26.0 \\
-71\end{array}$ & -6.3 & -1.5 & 4.5 & 1.1 & -36.2 & & \\
\hline 24261 & 1966-2012 & -1.7 & -1.8 & -1.2 & -4.5 & 5.3 & 8.1 & 17.9 & 6.5 & 2.0 & -4.2 & -0.3 & $\begin{array}{l}-3.5 \\
-67\end{array}$ & 32.6 & & \\
\hline 24266 & 1966-2015 & -2.9 & -2.9 & -1.3 & -3.1 & 2.2 & 5.8 & 3.0 & 5.1 & 7.3 & -3.9 & -1.9 & -5.0 & 9.0 & $\begin{array}{l}-19.3 \\
-36 \\
1979\end{array}$ & \\
\hline 24371 & $1966-2015$ & -1.3 & -1.7 & -0.7 & -4.6 & 1.8 & 13.9 & 5.7 & 8.5 & 5.5 & -4.8 & -2.5 & $\begin{array}{l}-4.2 \\
-63 \\
1985\end{array}$ & 10.8 & $\begin{array}{l}-23.6 \\
-46 \\
1996\end{array}$ & \\
\hline \multicolumn{17}{|c|}{ Indigirka River basin } \\
\hline 24382 & $1966-2015$ & $\begin{array}{l}-6.7 \\
-92 \\
1986\end{array}$ & \begin{tabular}{|l}
-4.8 \\
-71 \\
$\mathrm{~m}$
\end{tabular} & -2.9 & -1.0 & 4.3 & 5.2 & 3.3 & 7.1 & 4.1 & 0.0 & 0.8 & -4.3 & 11.8 & $\begin{array}{l}-16.0 \\
-29 \\
m\end{array}$ & \\
\hline 24585 & $1966-2015$ & $\begin{array}{l}-4.2 \\
-58\end{array}$ & -2.6 & 1.2 & -0.8 & -0.2 & 5.5 & -8.9 & 5.5 & 14.6 & 2.7 & 2.9 & -3.0 & 15.5 & & \\
\hline 24588 & $1966-2015$ & $\begin{array}{l}-4.3 \\
-68\end{array}$ & -1.5 & 0.0 & -2.8 & 1.7 & -12.1 & -8.8 & 2.8 & -0.2 & -1.7 & -1.7 & -1.9 & -26.6 & $\begin{array}{l}-14.9 \\
-26\end{array}$ & \\
\hline 24679 & 1966-2015 & $\begin{array}{l}-4.0 \\
-90\end{array}$ & $\begin{array}{l}-3.3 \\
-121\end{array}$ & -2.1 & -0.4 & 1.3 & $\begin{array}{l}20.7 \\
44 \\
\end{array}$ & -28.3 & -3.2 & 13.8 & -1.7 & -0.1 & -1.0 & 41.6 & $\begin{array}{l}-13.0 \\
-30\end{array}$ & \\
\hline 24684 & $1966-2015$ & -2.4 & -0.6 & 1.8 & 0.3 & -1.6 & 2.1 & -9.0 & -4.5 & 3.9 & 0.5 & 2.7 & -1.1 & 13.7 & & \\
\hline 24688 & $1966-2015$ & $\begin{array}{l}-4.4 \\
-60 \\
1980\end{array}$ & \begin{tabular}{|l|}
-3.0 \\
-44 \\
1994 \\
\end{tabular} & -1.3 & -0.6 & 0.5 & 7.5 & 0.1 & 9.7 & 8.5 & -1.0 & 3.0 & -4.5 & 40.9 & & $\begin{array}{l}53 \\
34 \\
\text { m } \\
\end{array}$ \\
\hline 24691 & $1966-2015$ & $\begin{array}{l}-5.5 \\
-62 \\
1987 \\
\end{array}$ & -3.6 & 1.1 & 1.7 & -3.5 & 9.2 & -2.8 & 12.3 & 5.8 & 3.0 & 3.5 & -3.8 & 15.9 & & \\
\hline 24076 & 1966-2012 & -2.0 & -2.6 & 1.6 & -3.8 & 3.1 & \begin{tabular}{|l|}
21.8 \\
49
\end{tabular} & 9.4 & 23.7 & $\begin{array}{l}15.3 \\
49\end{array}$ & 5.0 & 4.0 & -1.9 & 58.5 & & \\
\hline 21946 & 1966-2012 & -5.3 & -4.8 & 2.8 & 0.0 & -2.1 & -14.4 & $\begin{array}{l}-22.9 \\
-73 \\
\end{array}$ & -11.5 & -1.7 & 5.6 & 5.0 & 3.3 & -42.9 & & $\begin{array}{l}-48 \\
-60 \\
\end{array}$ \\
\hline
\end{tabular}


Table S3: Detected changes of monthly and annual streamflow ( $\mathrm{mm}$ and \%) and change point (year) and freshet onset dates. The Yana River basin

\begin{tabular}{|c|c|c|c|c|c|c|c|c|c|c|c|c|c|c|c|c|}
\hline ID & Period & $\begin{array}{l}\text { Area, } \\
\mathrm{km}^{2}\end{array}$ & Jan & Feb & Mar & Apr & May & Jun & Jul & Aug & Sep & Oct & Nov & Dec & Year & $\begin{array}{l}\text { Freshet } \\
\text { onset dates }\end{array}$ \\
\hline 3478 & $\begin{array}{l}1953- \\
2007\end{array}$ & 22.6 & NA & NA & NA & NA & 6.9 & 14.0 & 12.9 & $\begin{array}{l}13.0 \\
50 \\
1981 \\
\end{array}$ & $\begin{array}{l}7.1 \\
54 \\
\mathrm{~m} \\
\end{array}$ & $\begin{array}{l}0.12 \\
31 \\
1987 \\
\end{array}$ & 0.00 & 0.00 & $\begin{array}{l}79 \\
69 \\
1988 \\
\end{array}$ & 5.8 \\
\hline 3479 & $\begin{array}{l}1956- \\
2014\end{array}$ & 7570 & NA & NA & NA & NA & 5.6 & $\begin{array}{l}12.8 \\
64 \\
\mathrm{~m} \\
\end{array}$ & 4.6 & $\begin{array}{l}\mathbf{8 . 3} \\
\mathbf{5 4} \\
1987 \\
\end{array}$ & $\begin{array}{l}5.5 \\
67 \\
\mathrm{~m} \\
\end{array}$ & 0.1 & 0.0 & $\mathrm{NA}$ & 38 & \\
\hline 3474 & $\begin{array}{l}1949- \\
2007\end{array}$ & 8290 & NA & NA & NA & NA & $\begin{array}{l}7.5 \\
54 \\
1964 *\end{array}$ & -6.7 & $\begin{array}{l}-26 \\
-38 \\
m\end{array}$ & 8.7 & 8.4 & $\begin{array}{l}4.5 \\
78 \\
1991\end{array}$ & 0.3 & 0.0 & -7 & 10 \\
\hline 3424 & $\begin{array}{l}1957- \\
2015\end{array}$ & 16700 & 0.0 & $\mathrm{NA}$ & NA & NA & 2.9 & 7.3 & -5.5 & 0.4 & $\begin{array}{l}5.7 \\
53 \\
1982\end{array}$ & $\begin{array}{l}\mathbf{0 . 8} \\
\mathbf{5 4} \\
1987\end{array}$ & 0.1 & 0.0 & 9 & $\begin{array}{l}4.5 \\
\mathrm{~m}\end{array}$ \\
\hline 3430 & $\begin{array}{l}1956- \\
2015\end{array}$ & 23900 & 0.0 & NA & NA & NA & & 12.6 & -2.2 & 0.4 & $\begin{array}{l}7.6 \\
50 \\
1994 \\
\end{array}$ & $\begin{array}{l}2.0 \\
72 \\
1982 \\
\end{array}$ & $\begin{array}{l}0.4 \\
77 \\
1981 \\
\end{array}$ & $\begin{array}{l}0.1 \\
149 \\
1981 \\
\end{array}$ & 20 & \\
\hline 3483 & $\begin{array}{l}1945- \\
2015\end{array}$ & 40000 & 0.0 & 0.0 & NA & NA & $\begin{array}{l}3.6 \\
71 \\
1966^{*} \\
\end{array}$ & 3.4 & 1.8 & 9.0 & $\begin{array}{l}5.7 \\
46 \\
m \\
\end{array}$ & & & $\begin{array}{l}-0.04 \\
-54 \\
m \\
\end{array}$ & 24 & \\
\hline 3414 & $\begin{array}{l}1936- \\
2015\end{array}$ & 45300 & $\begin{array}{l}0.04 \\
161 \\
1977 \\
\end{array}$ & 0.0 & 0.0 & 0.0 & $\begin{array}{l}4.3 \\
60 \\
1965 * \\
\end{array}$ & 7.6 & 3.2 & 5.5 & $\begin{array}{l}5.8 \\
46 \\
1982 \\
\end{array}$ & $\begin{array}{l}1.0 \\
51 \\
1993 \\
\end{array}$ & $\begin{array}{l}0.3 \\
77 \\
1994 \\
\end{array}$ & $\begin{array}{l}0.1 \\
126 \\
1994 \\
\end{array}$ & $\begin{array}{l}24 \\
22 \\
m \\
\end{array}$ & $\begin{array}{l}6.5 \\
1978\end{array}$ \\
\hline 3443 & $\begin{array}{l}1960- \\
2015\end{array}$ & 52800 & 0.0 & NA & NA & 0.0 & $\begin{array}{l}15.5 \\
79 \\
1987\end{array}$ & $\begin{array}{l}18.6 \\
28 \\
1995\end{array}$ & $\begin{array}{l}17.4 \\
36 \\
1995\end{array}$ & $\begin{array}{l}27.0 \\
62 \\
1986 \\
\end{array}$ & $\begin{array}{l}19.0 \\
83 \\
1981\end{array}$ & $\begin{array}{l}3.3 \\
96 \\
1992\end{array}$ & $\begin{array}{l}0.2 \\
44 \\
1994\end{array}$ & 0.0 & $\begin{array}{l}104 \\
51 \\
1995\end{array}$ & $\begin{array}{l}\mathbf{6 . 8} \\
1995\end{array}$ \\
\hline 3445 & $\begin{array}{l}1937- \\
2015\end{array}$ & 89600 & 0.0 & 0.0 & 0.0 & 0.0 & $\begin{array}{l}12.4 \\
83 \\
1966 * \\
\end{array}$ & $\begin{array}{l}17.9 \\
28 \\
1988 \\
\end{array}$ & 7.4 & $\begin{array}{l}24.7 \\
63 \\
1982 \\
\end{array}$ & $\begin{array}{l}15.7 \\
69 \\
1981 \\
\end{array}$ & $\begin{array}{l}2.1 \\
54 \\
1992 \\
\end{array}$ & $\begin{array}{l}0.5 \\
54 \\
1987 \\
\end{array}$ & $\begin{array}{l}0.1 \\
75 \\
1987 \\
\end{array}$ & $\begin{array}{l}82 \\
42 \\
1987 \\
\end{array}$ & $\begin{array}{l}4.3 \\
\mathrm{~m}\end{array}$ \\
\hline 3861 & $\begin{array}{l}1972- \\
2007\end{array}$ & 224000 & $\begin{array}{l}0.03 \\
79 \\
1981 \\
\end{array}$ & 0.0 & $\begin{array}{l}-0.004 \\
-153 \\
\mathbf{1 9 8 9} \\
\end{array}$ & 0.0 & -0.8 & $\begin{array}{l}16.2 \\
40 \\
1983 \\
\end{array}$ & 9.4 & 9.2 & 7.8 & $\begin{array}{l}4.4 \\
118 \\
1993 \\
\end{array}$ & $\begin{array}{l}0.4 \\
118 \\
\text { m } \\
\end{array}$ & $\begin{array}{l}0.12 \\
75 \\
1982 \\
\end{array}$ & $\begin{array}{l}60 \\
40 \\
1983 \\
\end{array}$ & \\
\hline
\end{tabular}


Table S4: Detected changes of monthly and annual streamflow ( $\mathrm{mm}$ and \%) and change point (year) and freshet onset dates. The

\begin{tabular}{|c|c|c|c|c|c|c|c|c|c|c|c|c|c|c|c|c|}
\hline ID & Period & $\begin{array}{l}\text { Area, } \\
\mathrm{km}^{2}\end{array}$ & Jan & Feb & Mar & Apr & May & Jun & Jul & Aug & Sep & Oct & Nov & Dec & Year & $\begin{array}{c}\text { Freshet onset } \\
\text { dates }\end{array}$ \\
\hline 3516 & $\begin{array}{l}1964- \\
2014\end{array}$ & 16.6 & NA & NA & NA & 0.0 & $\begin{array}{l}\mathbf{2 5 . 7} \\
\mathbf{7 8} \\
1989 \\
\end{array}$ & 12.4 & -9.1 & 18.3 & $\begin{array}{l}36.8 \\
111 \\
1992 \\
\end{array}$ & $\begin{array}{l}1.6 \\
107 \\
1992\end{array}$ & 0.0 & 0.0 & $\begin{array}{l}115 \\
32 \\
1993\end{array}$ & \\
\hline 3527 & $\begin{array}{l}1945- \\
2014\end{array}$ & 23 & NA & NA & NA & NA & 3.9 & -11.1 & 10.9 & 6.4 & $\begin{array}{l}8.8 \\
87 \\
1993 \\
\end{array}$ & 0.0 & NA & NA & 21 & $\begin{array}{l}4.2 \\
\mathrm{~m}\end{array}$ \\
\hline 3510 & $\begin{array}{l}1946- \\
2014\end{array}$ & 644 & NA & NA & NA & NA & 5.2 & $\begin{array}{l}-14.2 \\
-61 \\
1967 *\end{array}$ & 5.3 & $\begin{array}{l}\mathbf{1 4 . 0} \\
\mathbf{6 5} \\
1982 \\
\end{array}$ & $\begin{array}{l}6.3 \\
61 \\
1993 \\
\end{array}$ & 0.1 & 0.0 & NA & 21 & \\
\hline 3499 & $\begin{array}{l}1956- \\
2015\end{array}$ & 7680 & NA & NA & NA & NA & & 11.6 & $\begin{array}{l}- \\
17.6\end{array}$ & -2.6 & $\begin{array}{l}9.9 \\
49 \\
1993 \\
\end{array}$ & $\begin{array}{l}3.3 \\
70 \\
1993 \\
\end{array}$ & $\begin{array}{l}0.43 \\
52 \\
1994 \\
\end{array}$ & 0.0 & 26 & $\begin{array}{l}5.4 \\
m\end{array}$ \\
\hline 3507 & $\begin{array}{l}1946- \\
2015\end{array}$ & 17600 & $\begin{array}{l}0.03 \\
59 \\
1985 \\
\end{array}$ & $\begin{array}{l}0.02 \\
91 \\
1985 \\
\end{array}$ & $\begin{array}{l}0.02 \\
86 \\
1985 \\
\end{array}$ & \begin{tabular}{|l|}
0.01 \\
57 \\
1985 \\
\end{tabular} & $\begin{array}{l}15.3 \\
106 \\
1964 *\end{array}$ & 4.0 & 2.9 & $\begin{array}{l}23.4 \\
53 \\
\text { m }\end{array}$ & \begin{tabular}{|l|l}
16.5 \\
76 \\
1993 \\
\end{tabular} & $\begin{array}{l}2.1 \\
61 \\
1990 \\
\end{array}$ & \begin{tabular}{|l|l}
0.6 \\
88 \\
1988 \\
\end{tabular} & $\begin{array}{l}0.1 \\
80 \\
1987 \\
\end{array}$ & $\begin{array}{l}81 \\
39 \\
1995 \\
\end{array}$ & $\begin{array}{l}7.6 \\
1967\end{array}$ \\
\hline 3518 & $\begin{array}{l}1945- \\
2015\end{array}$ & 22300 & 0.0 & NA & NA & NA & $\begin{array}{l}11.8 \\
90 \\
1966 * \\
\end{array}$ & -12.3 & -3.2 & 3.9 & $\begin{array}{l}9.1 \\
51 \\
1993 \\
\end{array}$ & $\begin{array}{l}0.9 \\
34 \\
1966 * \\
\end{array}$ & $\begin{array}{l}0.2 \\
47 \\
1994 \\
\end{array}$ & 0.0 & 14 & $\begin{array}{l}3.9 \\
1967\end{array}$ \\
\hline 3488 & $\begin{array}{l}1956- \\
2015\end{array}$ & 51100 & 0.1 & & & & $\begin{array}{l}7.6 \\
97 \\
1980 \\
\end{array}$ & 9.9 & -4.4 & 7.6 & $\begin{array}{l}12.5 \\
59 \\
1993 \\
\end{array}$ & & & 0.0 & 43 & $\begin{array}{l}5.1 \\
\mathrm{~m}\end{array}$ \\
\hline 3489 & $\begin{array}{l}1944- \\
2015\end{array}$ & 83500 & & $\begin{array}{l}0.10 \\
86 \\
1977 \\
\end{array}$ & $\begin{array}{l}0.10 \\
120 \\
1983 \\
\end{array}$ & \begin{tabular}{|l}
0.14 \\
159 \\
1981 \\
\end{tabular} & $\begin{array}{l}7.4 \\
92 \\
1966 * \\
\end{array}$ & -0.6 & -6.0 & $\begin{array}{l}13.2 \\
34 \\
\text { m } \\
\end{array}$ & \begin{tabular}{|l|l}
11.4 \\
55 \\
1993 \\
\end{tabular} & $\begin{array}{l}1.4 \\
34 \\
1993 \\
\end{array}$ & $\begin{array}{l}0.4 \\
28 \\
1982 \\
\end{array}$ & \begin{tabular}{|l|l}
0.3 \\
63 \\
1983 \\
\end{tabular} & $\begin{array}{l}33 \\
19 \\
1995 \\
\end{array}$ & $\begin{array}{l}\mathbf{4 . 6} \\
1967\end{array}$ \\
\hline 3871 & $\begin{array}{l}1936- \\
1996\end{array}$ & $\begin{array}{l}30500 \\
0\end{array}$ & $\begin{array}{l}-0.08 \\
-25 \\
1964 * \\
\end{array}$ & -0.01 & 0.01 & 0.01 & 0.2 & 0.3 & -6.3 & 4.3 & $\begin{array}{l}11 \\
49 \\
1965 \\
\end{array}$ & 0.7 & -0.1 & $\begin{array}{l}-0.2 \\
-32 \\
1969 \\
\end{array}$ & 8.1 & \\
\hline
\end{tabular}


Table S5: Detected changes of maximum water discharges in May - September $\left(\mathrm{m}^{3} \mathrm{~s}^{-1}\right.$ and $\%)$ and change points (year).

Only those gauges are included into the table, where the changes were identified.

\begin{tabular}{|c|c|c|c|c|c|}
\hline ID & May & June & Jul & Aug & Sep \\
\hline 3516 & & & & & $\begin{array}{l}0.71 \\
65.5 \\
1993\end{array}$ \\
\hline 3527 & & & & & $\begin{array}{c}0.22 \\
54.1 \\
1965,1971\end{array}$ \\
\hline 3510 & & $\begin{array}{c}-13.5 \\
-47.7 \\
m\end{array}$ & & $\begin{array}{l}17.5 \\
69.9 \\
1982 \\
\end{array}$ & \begin{tabular}{|c|}
5.3 \\
47.4 \\
1993 \\
\end{tabular} \\
\hline 3499 & $\begin{array}{c}62.0 \\
64.8 \\
m\end{array}$ & & & & $\begin{array}{c}63.4 \\
43.3 \\
\mathrm{~m} \\
\end{array}$ \\
\hline 3507 & $\begin{array}{l}431 \\
83.4 \\
1964 \\
\end{array}$ & & & & $\begin{array}{c}298 \\
78.3 \\
1981,1993\end{array}$ \\
\hline 3518 & $\begin{array}{c}403 \\
72.0 \\
1987,1966 \\
\end{array}$ & & & & $\begin{array}{c}183 \\
44.8 \\
1971,1993 \\
\end{array}$ \\
\hline 3488 & $\begin{array}{c}384 \\
59.4 \\
1987,1984 \\
\end{array}$ & & & & $\begin{array}{l}445 \\
58.7 \\
1993\end{array}$ \\
\hline 3489 & $\begin{array}{l}848 \\
71.2 \\
1965 \\
\end{array}$ & & & & $\begin{array}{c}794 \\
58.9 \\
1973,1993 \\
\end{array}$ \\
\hline 3478 & & & $\begin{array}{c}0.85 \\
84.4 \\
\mathrm{~m} \\
\end{array}$ & & \\
\hline 3479 & & \begin{tabular}{|l|}
163 \\
81.8 \\
1994 \\
\end{tabular} & & $\begin{array}{c}96 \\
62.6 \\
1987 \\
\end{array}$ & $\begin{array}{c}49 \\
68.2 \\
\mathrm{~m}\end{array}$ \\
\hline 3424 & & & & & $\begin{array}{c}93.6 \\
53.3 \\
m\end{array}$ \\
\hline 3430 & & & & & $\begin{array}{c}116 \\
36.4 \\
m\end{array}$ \\
\hline 3483 & & & & & $\begin{array}{l}158 \\
31.8\end{array}$ \\
\hline 3414 & $\begin{array}{l}495 \\
85.2 \\
1964 \\
\end{array}$ & & & & $\begin{array}{c}239 \\
50.3 \\
1976,1995 \\
\end{array}$ \\
\hline 3443 & & & $\begin{array}{c}1100 \\
46.7 \\
\mathrm{~m}\end{array}$ & $\begin{array}{c}1021 \\
48.8 \\
\mathrm{~m}\end{array}$ & $\begin{array}{l}815 \\
70.5 \\
1981 \\
\end{array}$ \\
\hline 3445 & $\begin{array}{c}1520 \\
65.6 \\
1988,1966 \\
\end{array}$ & & & $\begin{array}{c}1631 \\
55.8 \\
1986,1984 \\
\end{array}$ & $\begin{array}{l}985 \\
59.8 \\
1981 \\
\end{array}$ \\
\hline 3861 & & & & $\begin{array}{l}1298 \\
26.4 \\
1984\end{array}$ & \\
\hline
\end{tabular}




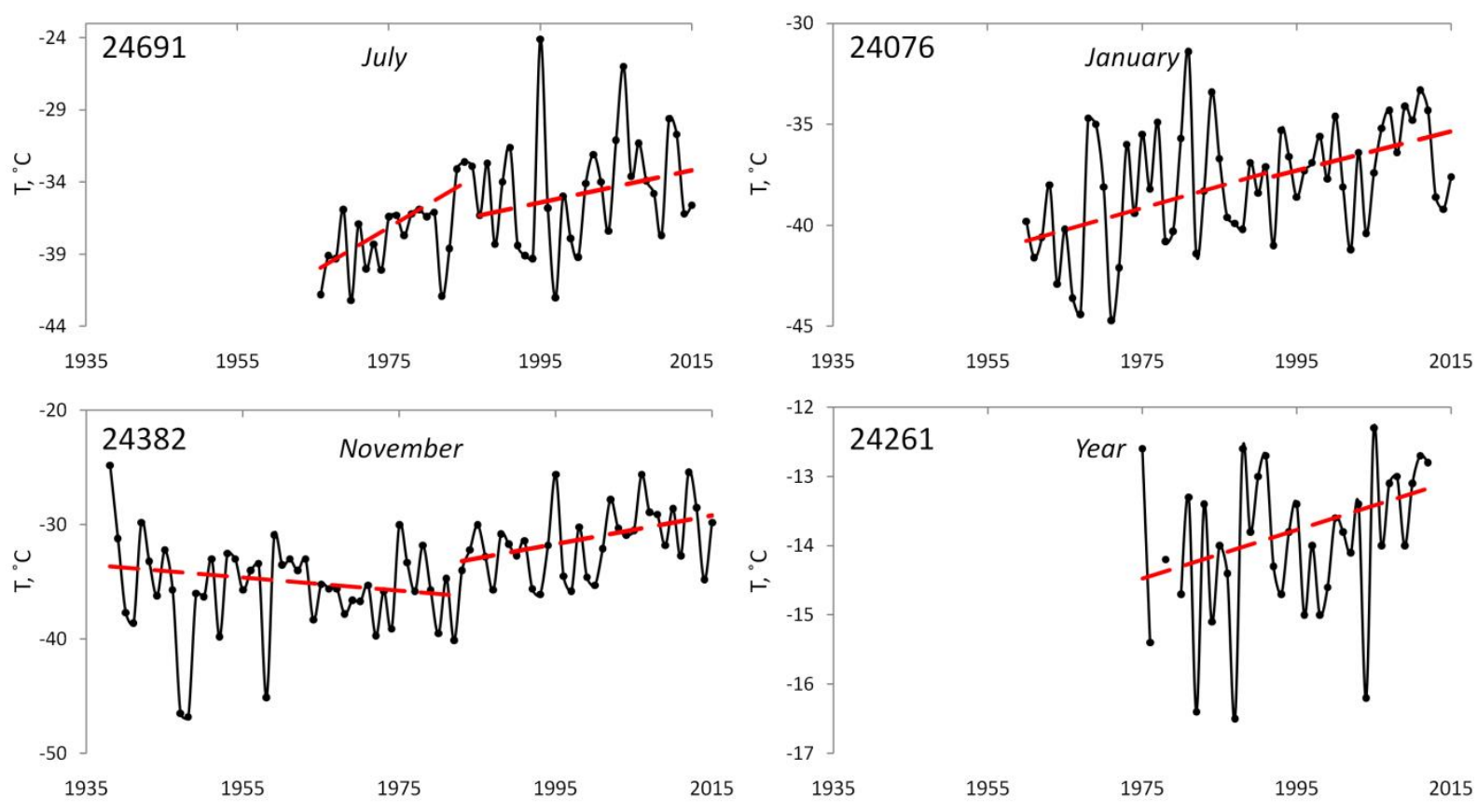

Fig. S1 Changes of air temperature at some meteorological stations of the studied region
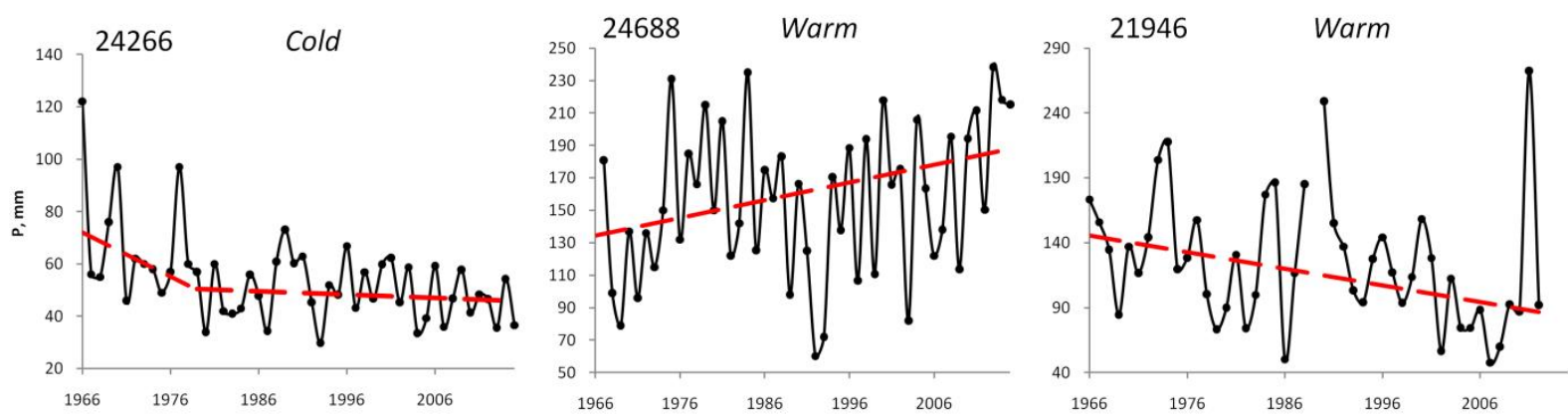

Figure S2: Changes of precipitation at some meteorological stations of the studied region 

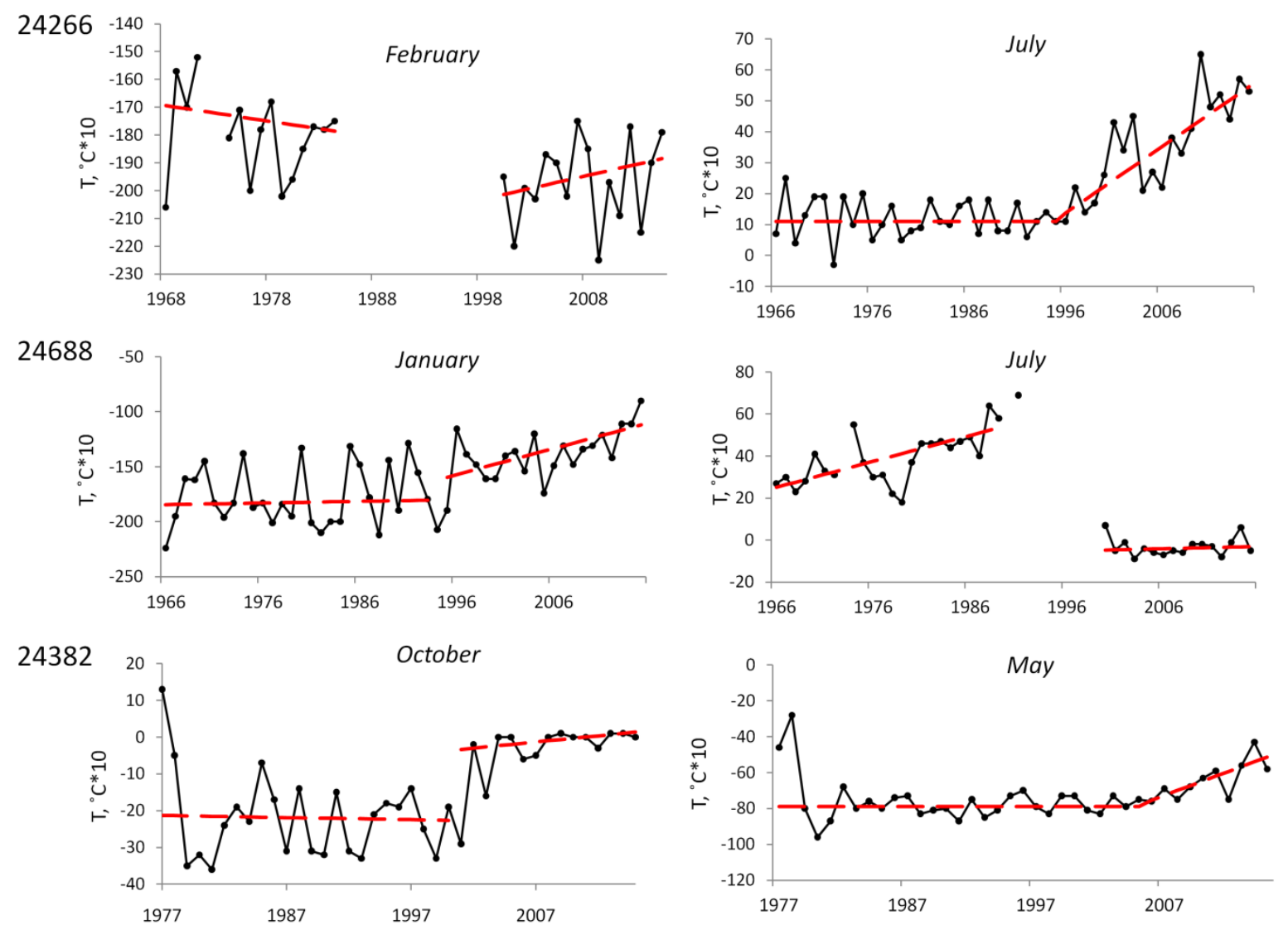

Figure S3: Changes of monthly soil temperature at $80 \mathrm{~cm}$ depth at the Verkhoyansk (ID24266), Oymyakon (ID24688) and Ust'-Moma (ID24382) meteorological stations, 1966 (1977)-2015. Red dash line is Sen's estimate. The year of change point where continuous data is available was estimated with the Pettitt's test at $p \leq 0.05$
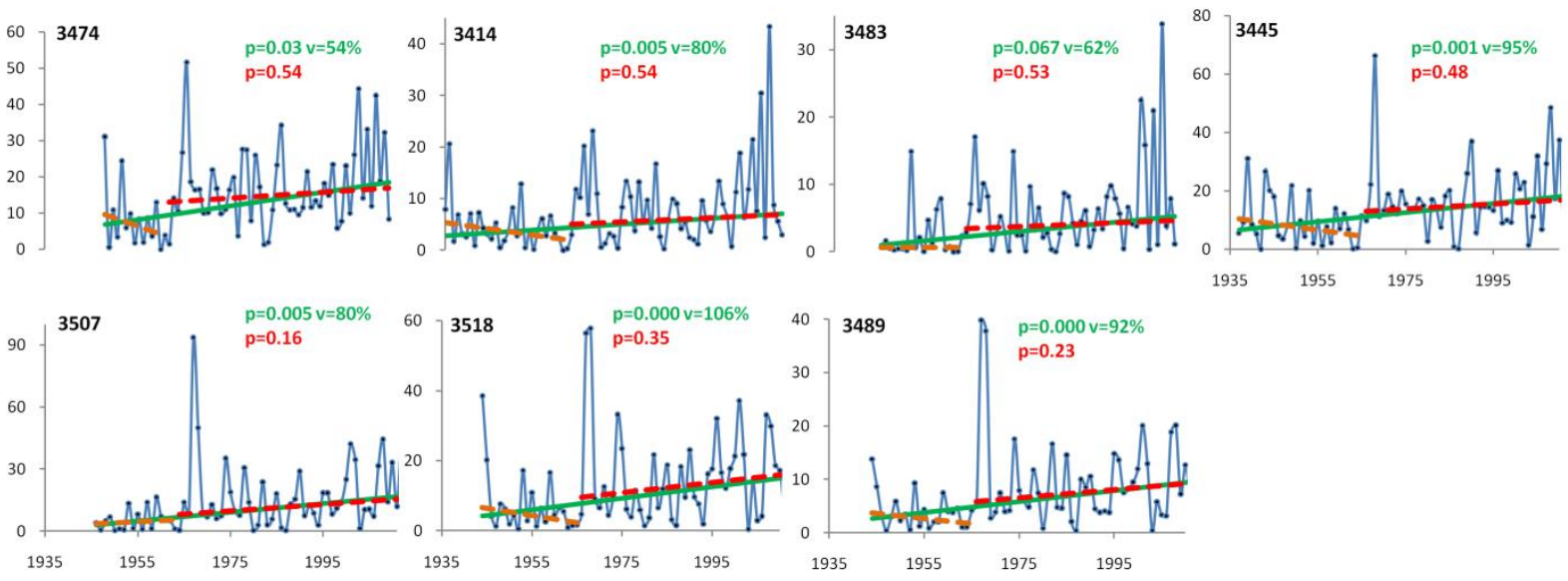

Figure S4: Patterns of streamflow changes in May, group A (green, orange and red - the trend slope over the period of record, before and after change point respectively) 

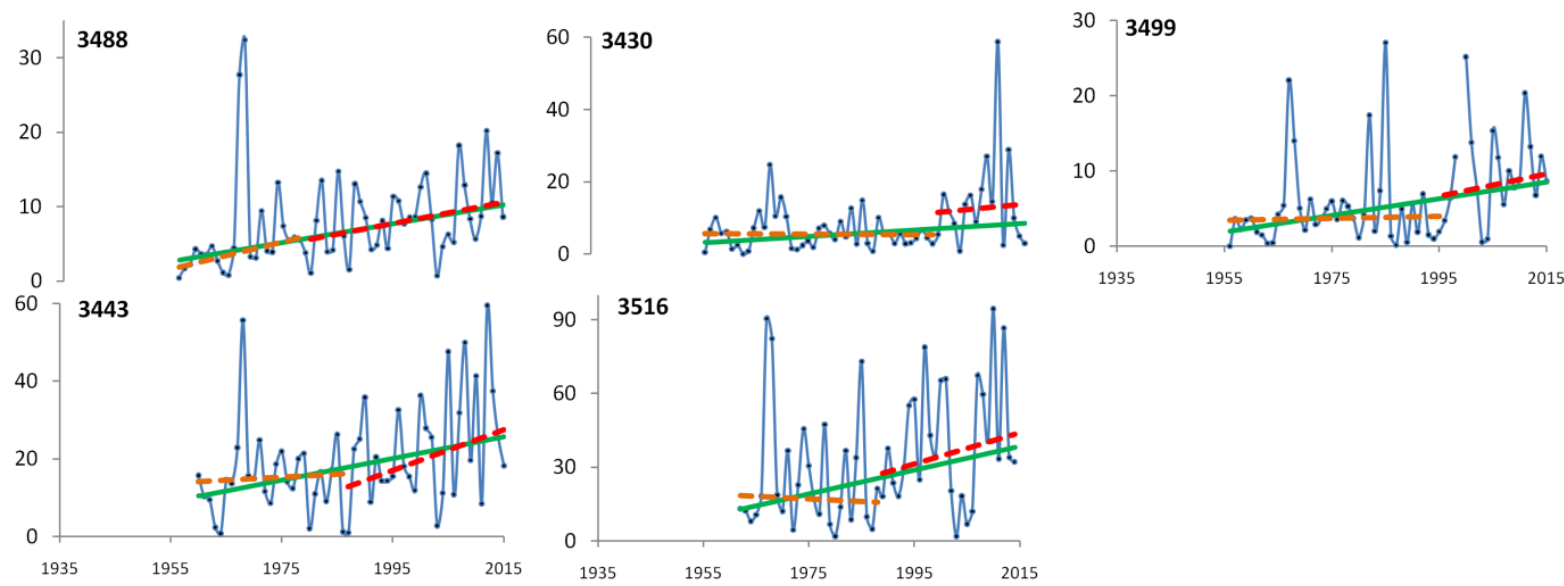

50 Figure S5: Patterns of streamflow changes in May, group B (green, orange and red - the trend slope over the period of record, before and after change point respectively)
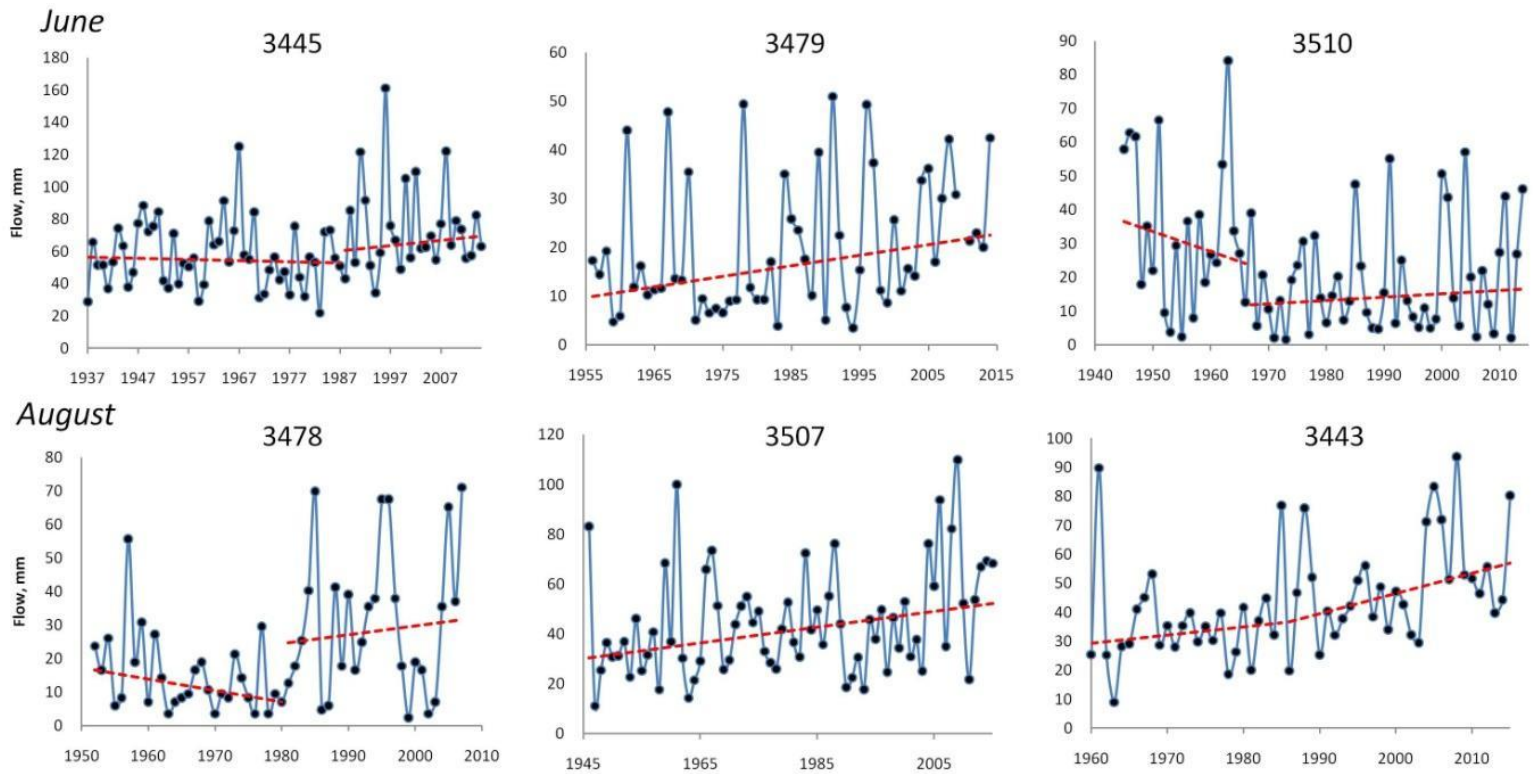

Figure S6: Changes of streamflow in June and August 


\section{September}
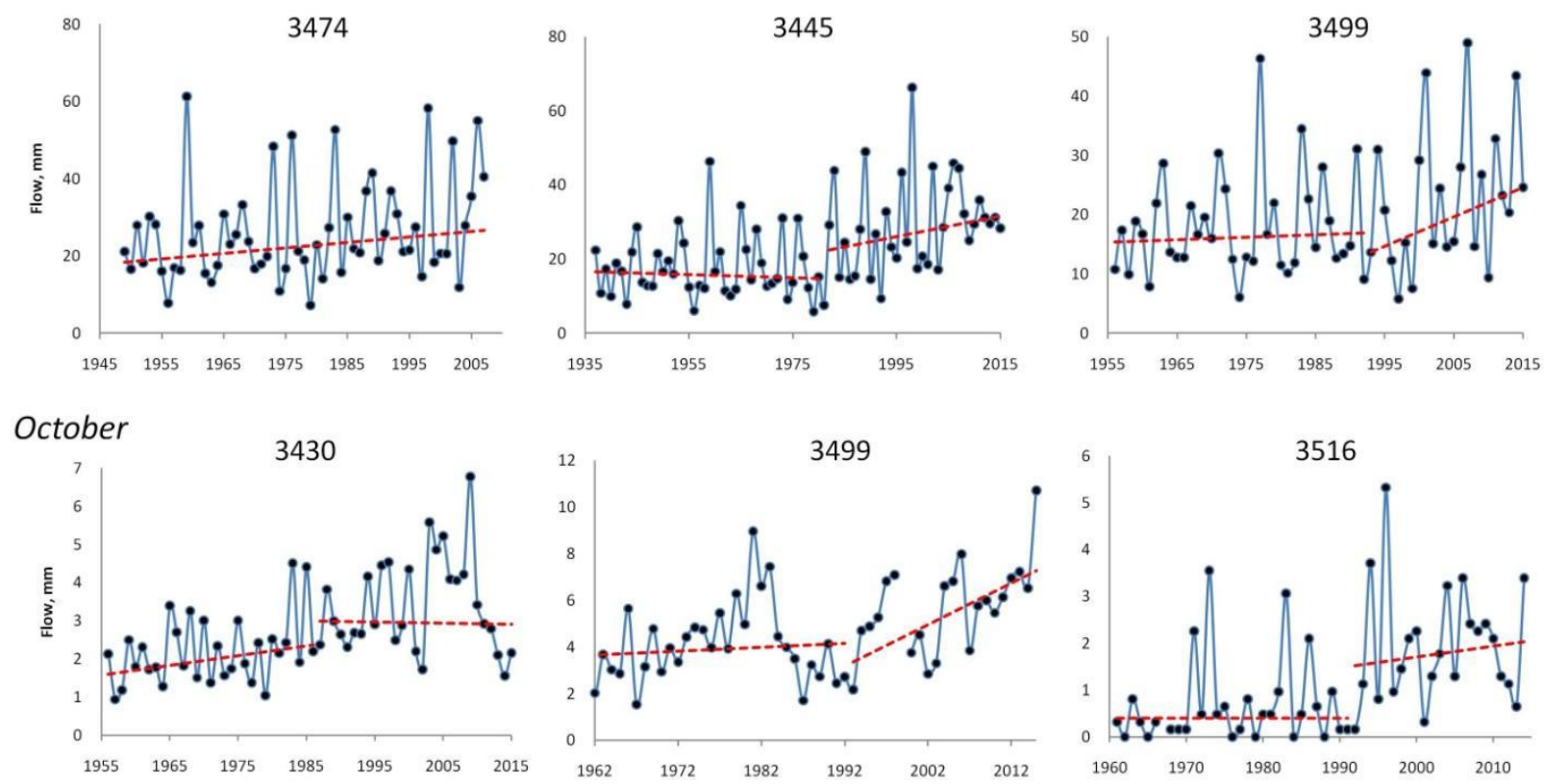

Figure S7: Changes of streamflow in September and October
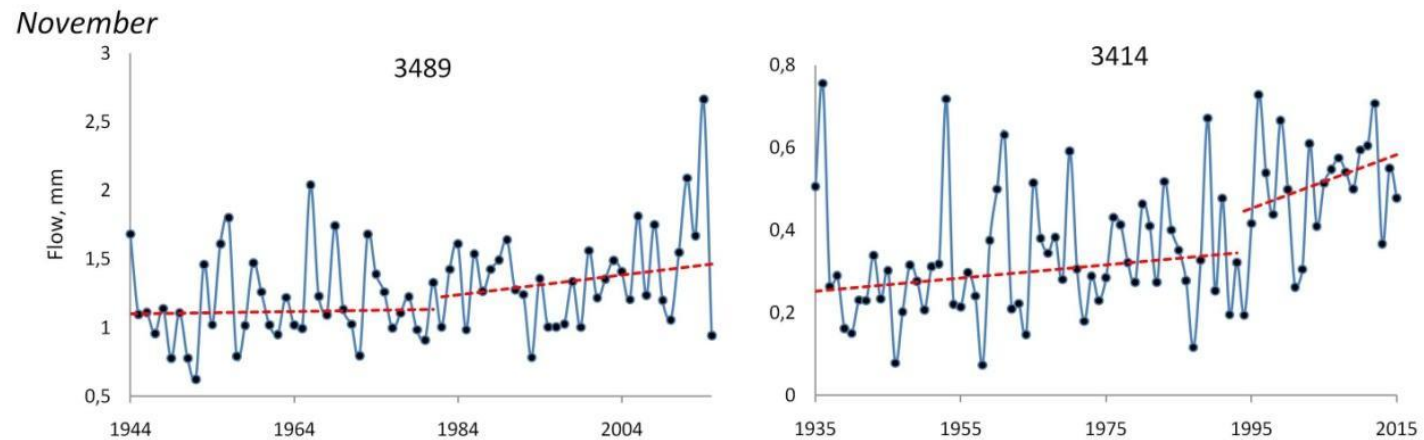

December
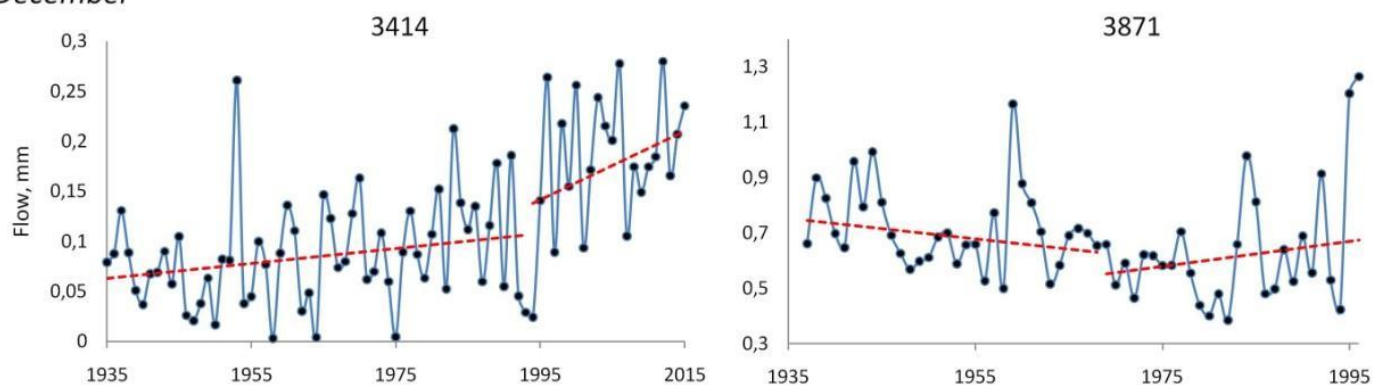

Figure S8: Changes of streamflow in November and December 

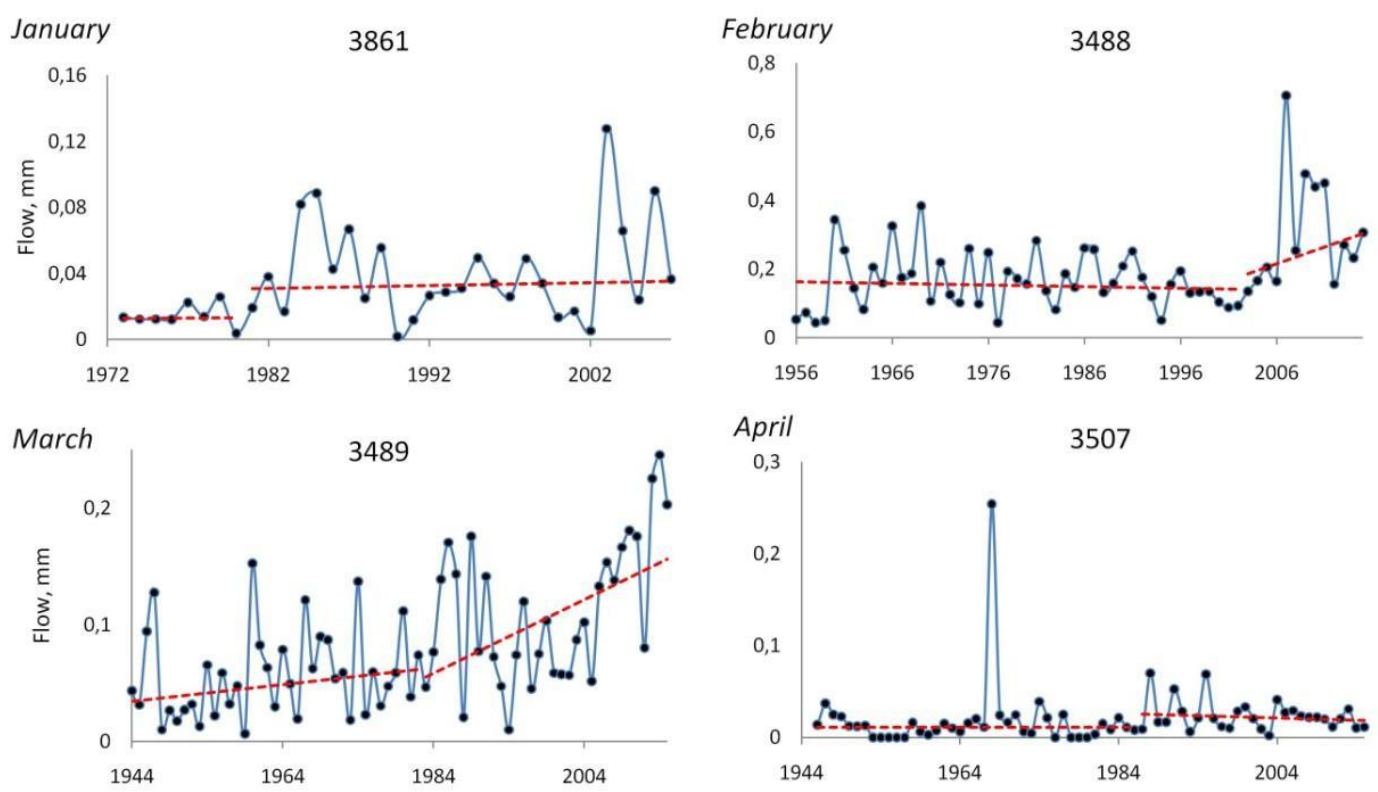

60 Figure S9: Changes of streamflow in January - April
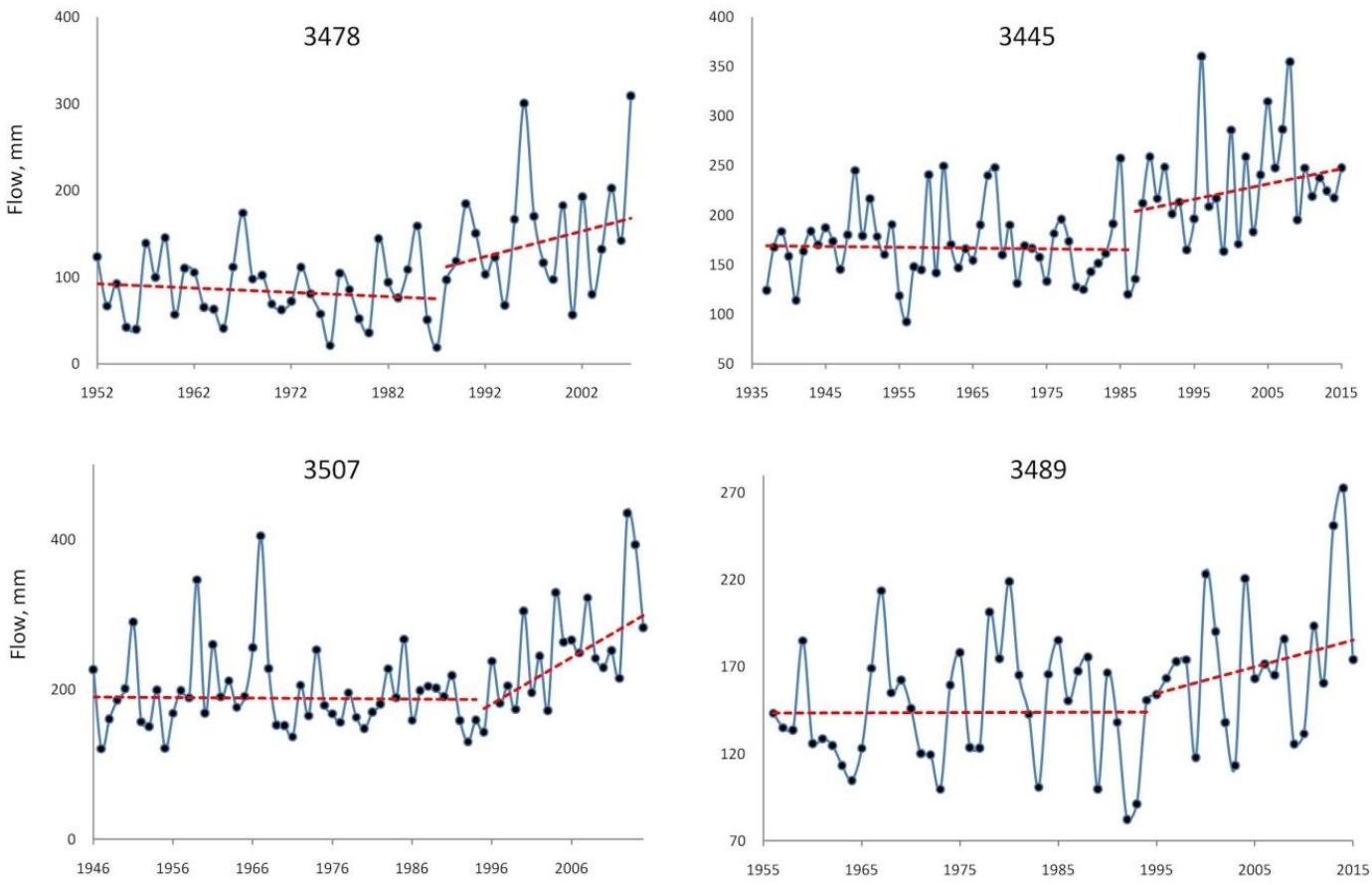

Figure S10: Changes of annual streamflow 


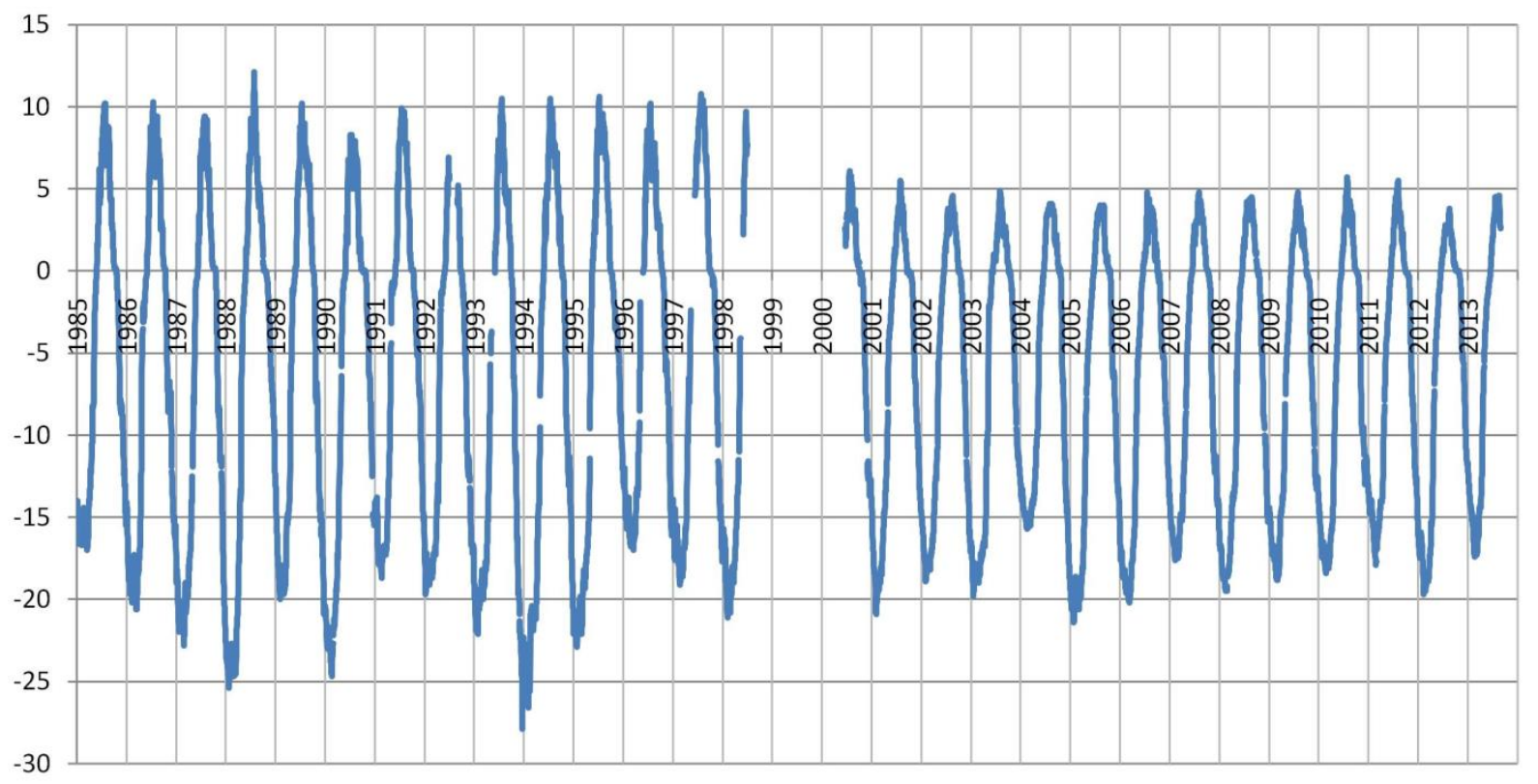

65 Figure S11: Soil temperature at Oymyakon (ID 24688) at $0.4 \mathrm{~m}$ depth, 1985-2013. One may note abrupt change of maximum soil temperature after data gap in 1999-2000.

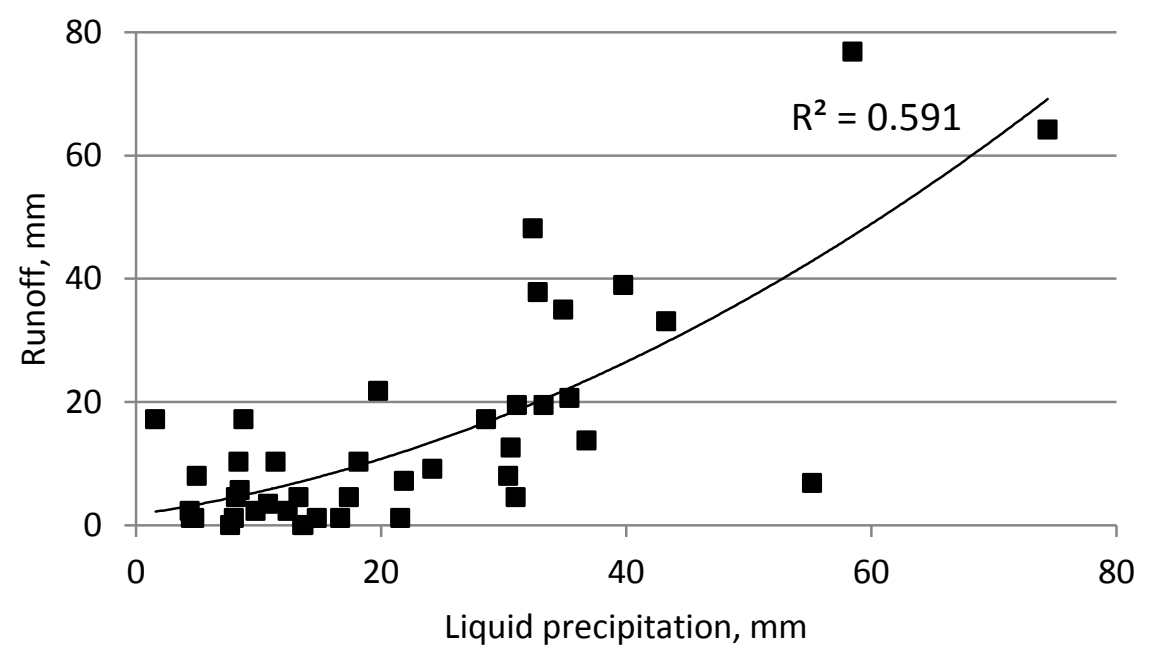

Figure S12: Correlation of runoff and liquid precipitation in September, Ust-Charky meteorological station (ID 24371) - gauge ID 3478 


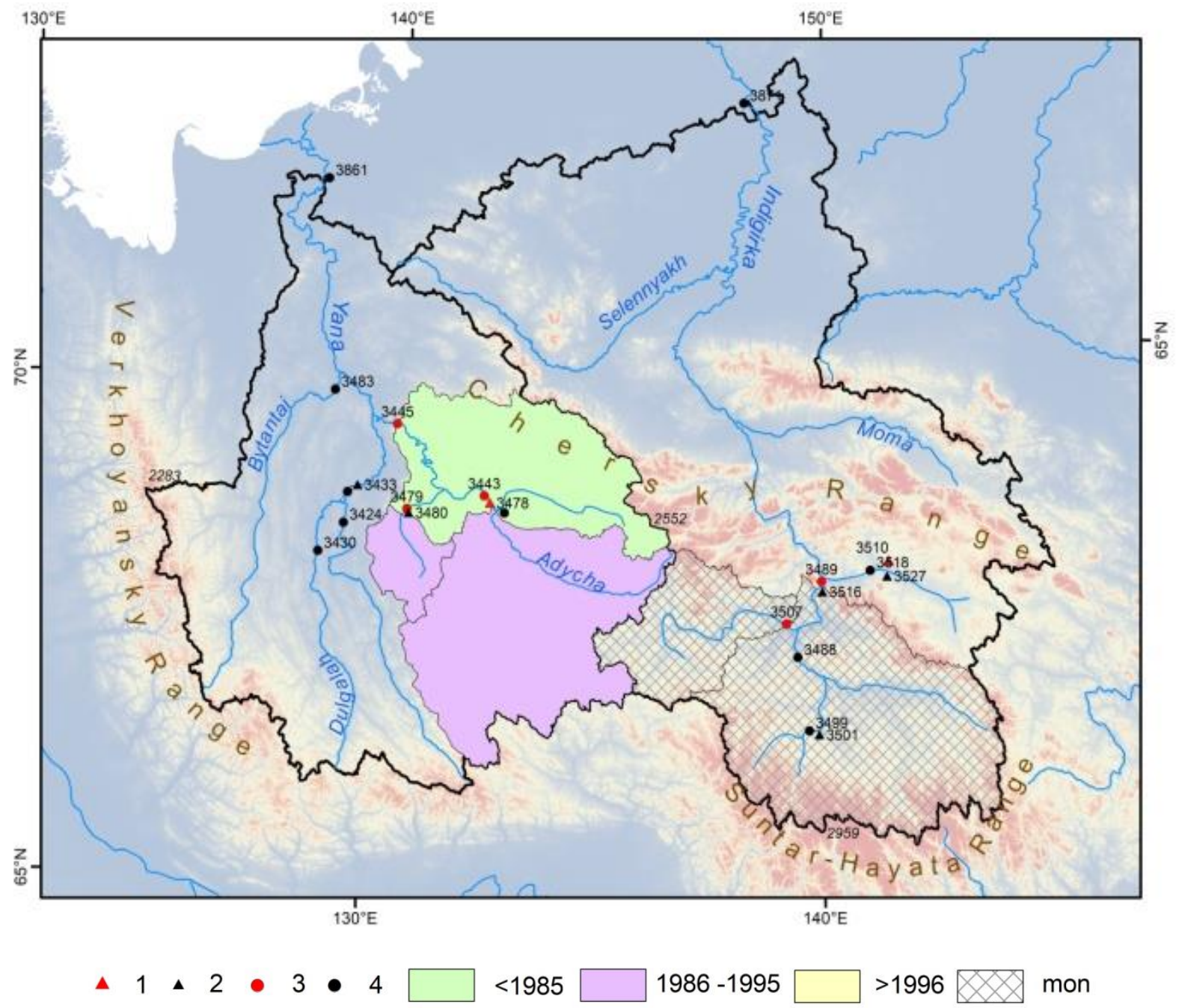

75 Figure S13: Changes of streamflow in August. Here and in following Fig. S14 - S18: statistically significant trends values are divided into 4 groups and painted with different colours accordingly: change points $<1985$ - green, 1986-1995 - violet, 1996 and later yellow, check - monotonically trends. 1 - gauges with significance changes (basin area < $1000 \mathrm{~km}^{2}$ ), 2 - gauges without significance changes (basin area $<1000 \mathrm{~km}^{2}$ ), 3 - gauges

80 with significance changes (basin area $>1000 \mathbf{k m}^{2}$ ), 2 - gauges without significance changes (basin area $>1000 \mathrm{~km}^{2}$ ) 


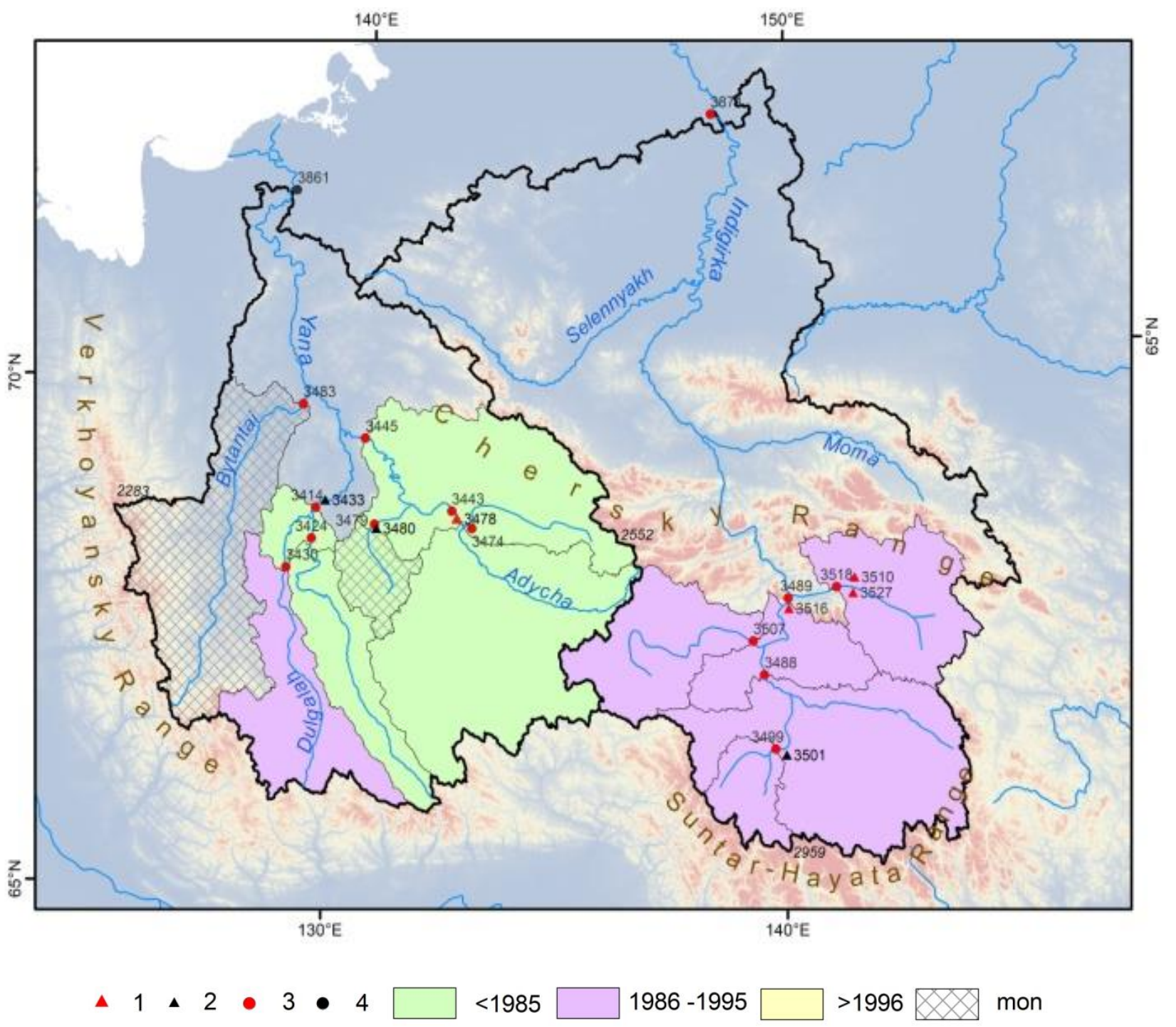

Figure S14: Changes of streamflow in September. All designations are the same as in Fig. S13. 


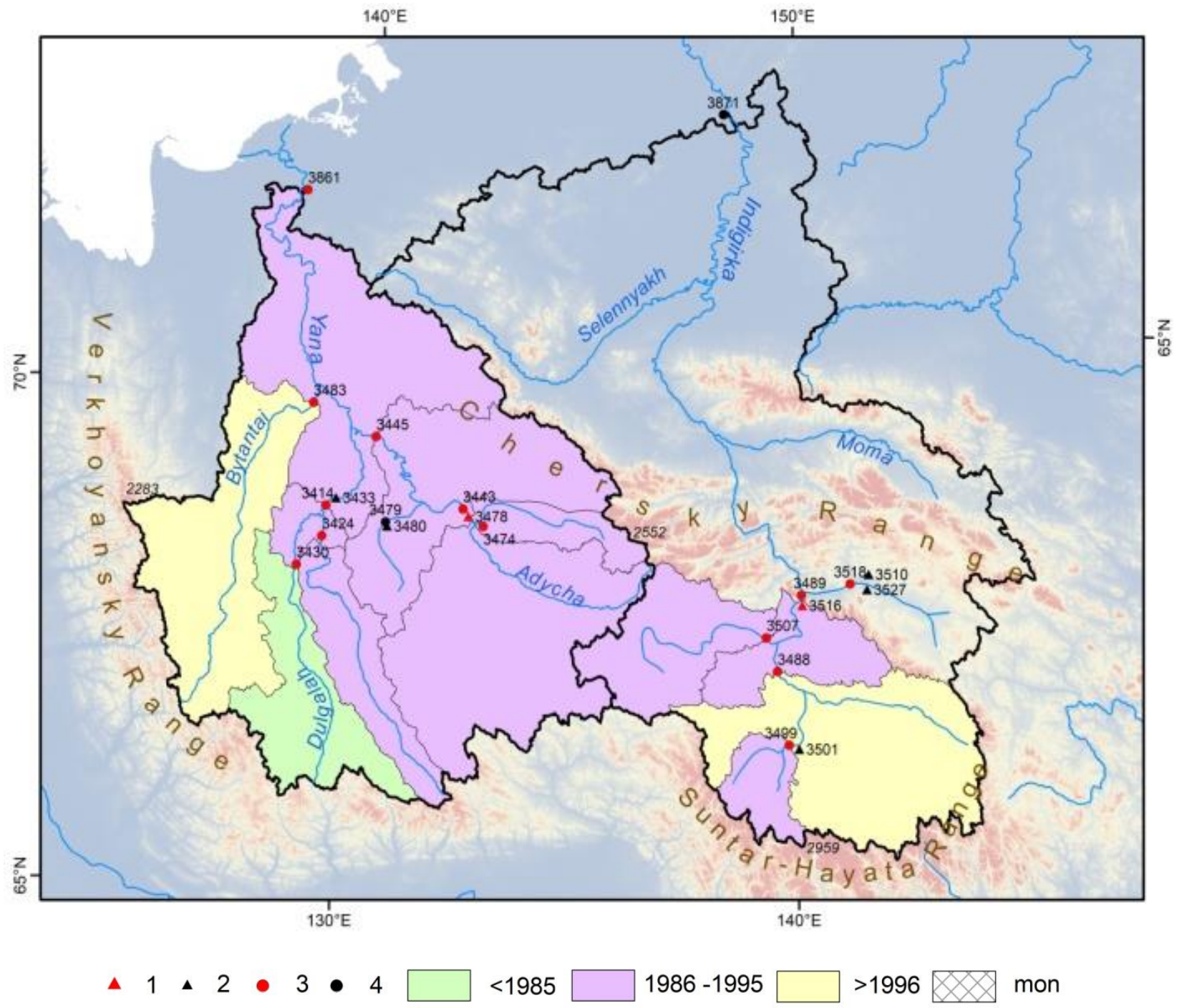

Figure S15: Changes of streamflow in October. All designations are the same as in Fig. S13 

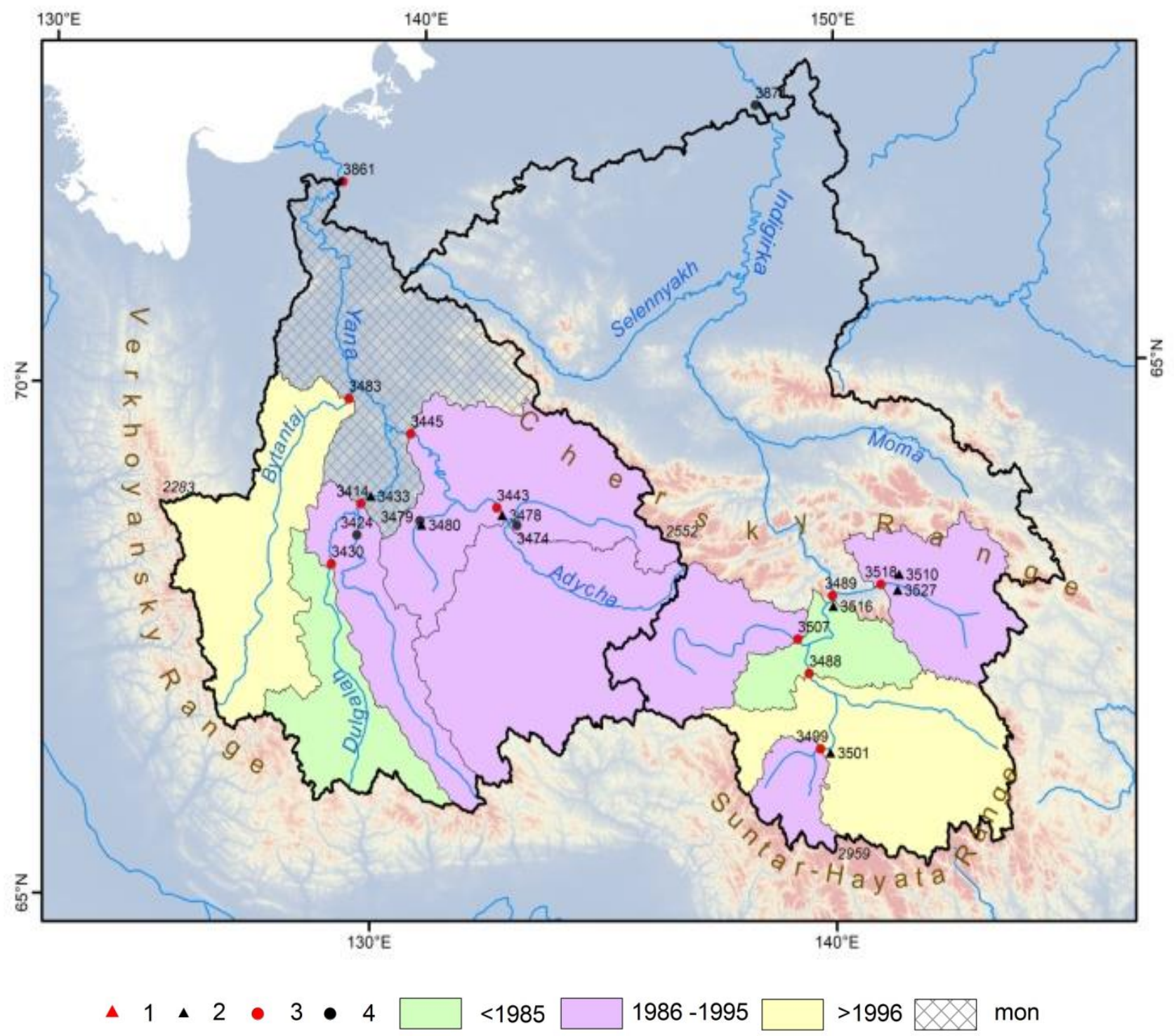

Figure S16: Changes of streamflow in November. All designations are the same as in Fig. S13. 


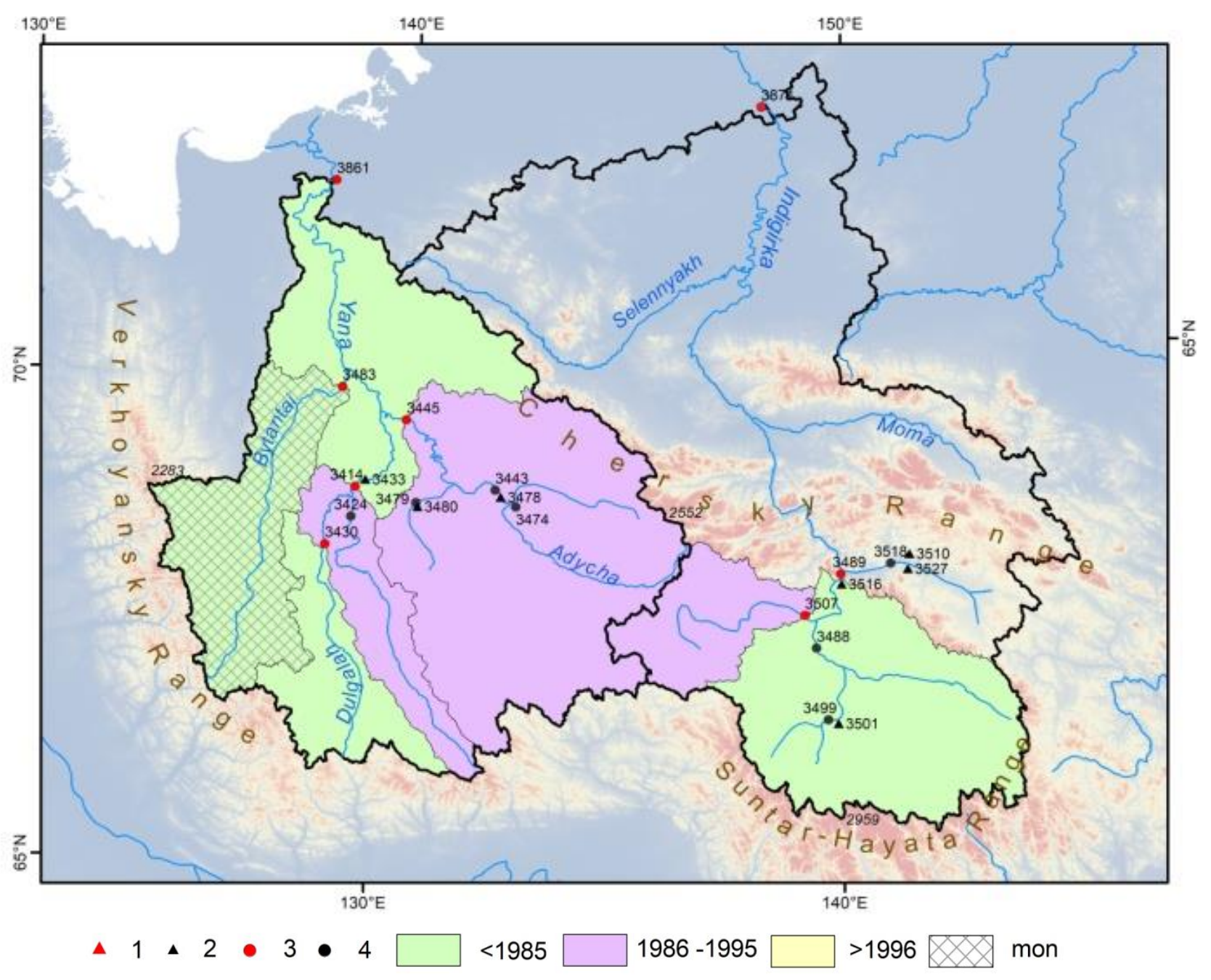

Figure S17: Changes of streamflow in December. All designations are the same as in Fig. S13. 


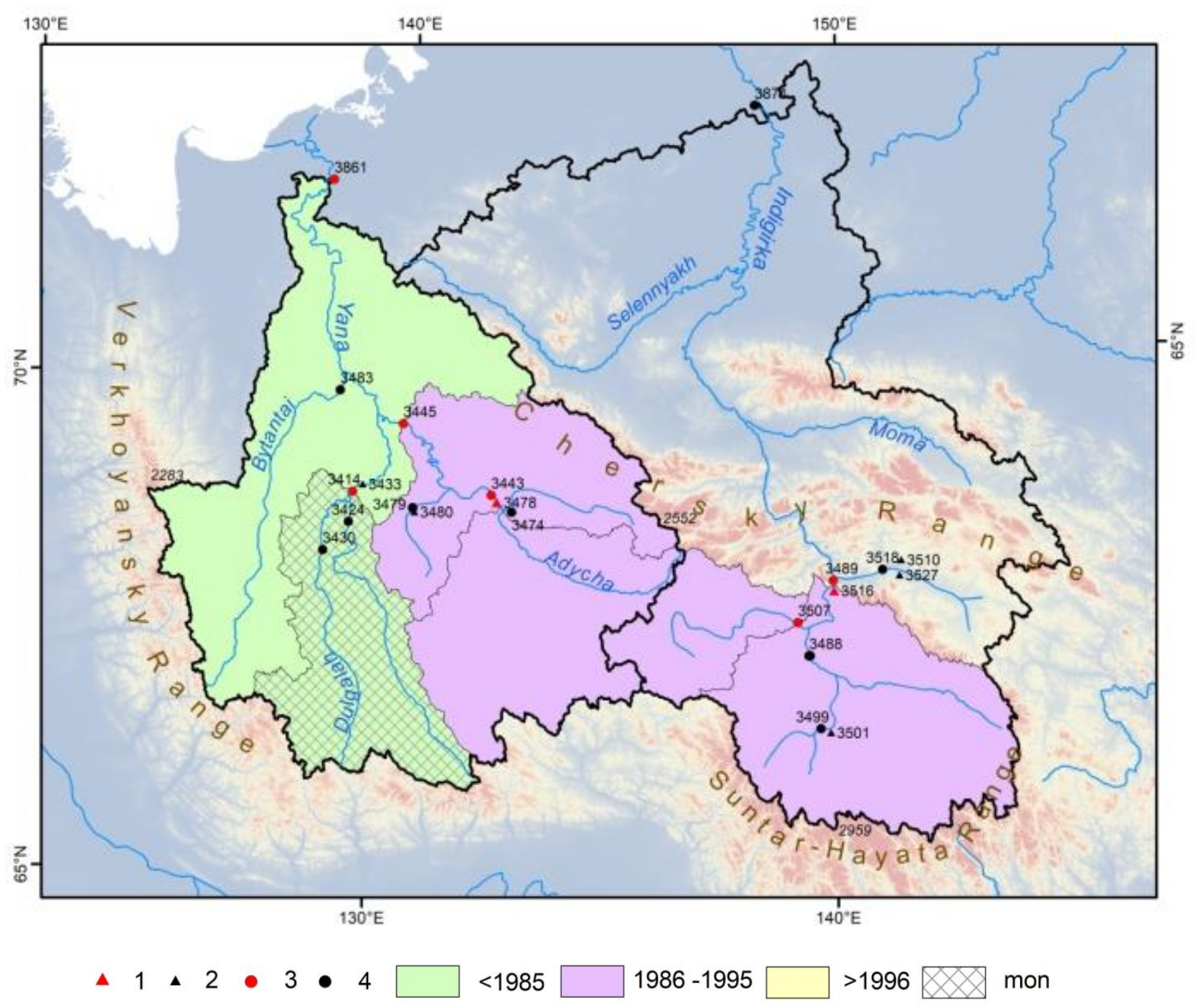

Figure S18: Changes of annual streamflow. All designations are the same as in Fig. S13. 